\title{
Elliptic blending model: A new near-wall Reynolds-stress turbulence closure
}

\author{
Rémi Manceau ${ }^{\text {a) }}$ and Kemal Hanjalic \\ Department of Applied Physics, Thermofluids Section, Delft University of Technology, Lorentzweg 1, \\ P.O. Box 5046, 2600 GA Delft, The Netherlands
}

(Received 19 December 2000; accepted 14 November 2001)

\begin{abstract}
A new approach to modeling the effects of a solid wall in one-point second-moment (Reynolds-stress) turbulence closures is presented. The model is based on the relaxation of an inhomogeneous (near-wall) formulation of the pressure-strain tensor towards the chosen conventional homogeneous (far-from-a-wall) form using the blending function $\alpha$, for which an elliptic equation is solved. The approach preserves the main features of Durbin's Reynolds-stress model, but instead of six elliptic equations (for each stress component), it involves only one, scalar elliptic equation. The model, called "the elliptic blending model," offers significant simplification, while still complying with the basic physical rationale for the elliptic relaxation concept. In addition to model validation against direct numerical simulation in a plane channel for $\operatorname{Re}_{\tau}=590$, the model was applied in the computation of the channel flow at a "real-life" Reynolds number of $10^{6}$, showing a good prediction of the logarithmic profile of the mean velocity. (C) 2002 American Institute of Physics. [DOI: 10.1063/1.1432693]
\end{abstract}

\section{INTRODUCTION}

Modeling the effects of solid walls on adjacent turbulent flows has long been-and still is - a major challenge. The problem is equally acute in one-point and two-point statistical closures, as it is in spectral modeling or large-eddy simulations (LES). Indeed, the hypotheses underlying existing one-point turbulence closure models, e.g., high Reynolds number, local isotropy, quasihomogeneity, are not valid in the presence of a wall. Hence, near-wall modifications are necessary in order to make them comply with the near-wall behavior of turbulence.

Research on this topic is driven by two opposing motivations: a need for simple and convenient models for industrial applications, and the requirement for consistency with the physics of the near-wall turbulence. The wall-function technique ${ }^{1}$ is widely used among the industry because it enables a drastic reduction in the number of grid points, but is still deficient in nonequilibrium flows, primarily in strong pressure gradients, impinging flows, separation, reattachment, natural convection, three-dimensional flows, etc. Models based on damping functions, which allow the integration of equations up to the wall, are much less popular among the industry since they require a very fine mesh in the vicinity of the wall and introduce nonlinear (typically exponential) functions in the equations, which make their solution more difficult. Moreover, most of these functions are purely empirical and lack theoretical justification. More elaborate models (e.g., Lumley, ${ }^{2}$ Shih and Lumley, ${ }^{3}$ Craft and Launder ${ }^{4}$ ),

\footnotetext{
a) Author to whom correspondence should be addressed. Present address: Laboratoire d'Etudes Aérodynamiques, Université de Poitiers, SP2MI, Téléport 2, Bd Marie et Pierre Curie, BP 30179, 86962 Futuroscope Chasseneuil Cedex, France; electronic mail: Remi.Manceau@lea.univpoitiers.fr
}

based on constitutive relations and physical constraints such as realizability or two-component limit of turbulence, have better theoretical bases but are very seldom-if at all-used for industrial applications because of their complexity.

The elliptic relaxation method of Durbin ${ }^{5}$ offers good prospects of reconciling the two above-mentioned requirements: it enables the integration down to the wall, with acceptable grid density. The method, applied to Reynoldsstress models, has a solid theoretical basis, but implies six additional equations, which impedes its spreading into the industry. The main problem is not the increased cost due to the number of equations, but rather the complexity of the implementation and the stability problems: the boundary conditions for the additional equations are a major source of numerical instability. For industrial applications, Durbin proposed a version of the model reduced to four equations, the $\overline{v^{2}}-f$ model, ${ }^{6}$ which has become popular and has begun to be implemented in commercial software. However, this model is not fully satisfactory because it still uses the eddy viscosity hypothesis.

\section{A. The physics of wall effects on turbulence}

A solid wall exerts multiple effects on fluid flow and turbulence. The no-slip constraint imposes the dominating role of fluid viscosity in the close vicinity of a wall regardless of the bulk-flow Reynolds number. Viscous effects are of scalar character and dampen the velocity fluctuations equally in all directions. In contrast, the blocking effect originating from the impermeability constraint suppresses the velocity fluctuations primarily in the wall-normal direction, making the turbulence highly anisotropic and, in the limit, forces the turbulence to approach the two-component state at the edge of the viscous sublayer. In addition, the wall reflects the pres- 
sure fluctuations and enhances their scrambling effect, thus redistributing the kinetic energy among the components, which, in turn, leads to the reduction of the turbulence anisotropy. The latter two (opposing) effects, which have a directional orientation and depend on the wall topography, are of a kinematic character and are present also in the vicinity of an interface (free surface) between a liquid and gaseous fluid. A physically consistent model ought to account separately for each of the effects mentioned, something that is difficult to achieve with a limited number of flow and turbulence parameters that are at one's disposal in one-point turbulence models, regardless of their level.

Most models of near-wall turbulence do not distinguish the viscous from nonviscous effects and usually apply empirical damping functions in terms of local turbulence Reynolds numbers and often of wall distance by which to account for the total wall effect. Needless to say, such models cannot perform well in situations where one or the other effect is absent or is of less importance (e.g., viscous and transitional regions in flows away from a solid wall unaffected by blocking, or flows with liquid-gas interface where the kinematic blocking is the sole cause of turbulence modification).

Several approaches to model viscous and nonviscous wall effects separately have been reported in the literature. Craft and Launder ${ }^{4}$ apply nonlinear models for the pressurestrain term in which the coefficients are determined by imposing-among others-the two-component turbulence limit, which is the major consequence of wall blockage, while also introducing some functions to model the viscous effects. Hanjalic et al. $^{7}$ model the viscous effects with functions of turbulence Reynolds number defined solely in terms of turbulence kinetic energy and its dissipation rate (hence invariant). Recognizing the fact that the turbulence anisotropy in the near-wall region is primarily caused by wall blockage, the turbulent-stress and dissipation-rate anisotropy invariants are used to model this nonviscous effect. Durbin's concept of elliptic relaxation, both in the eddy-viscosity and Reynolds-stress models, accounts in fact for the kinematic wall blocking, which adjusts the wall effect on pressureredistribution, stress anisotropy, and stress dissipation rates, while the viscous effects are introduced by imposing the Kolmogorov scales as the lower bounds to the conventional large-eddy time and length scales.

\section{B. The present contribution}

We propose to reduce the number of equations in Durbin's Reynolds-stress model and thus to reduce the complexity of the model. Moreover, one of the main purposes of this modification is to suppress the previously mentioned numerical stiffness induced by the boundary conditions of the additional elliptic relaxation equations. It is aimed to meet industrial needs for a simple and robust model, while still preserving the elliptic relaxation concept and satisfying the main theoretical constraints pertinent to near-wall turbulence.

It is first noted that the six elliptic relaxation equations are somewhat redundant. Indeed, in this model, the redistri- bution term $\phi_{i j}^{*}$ is evaluated from $k f_{i j}$, where $k$ is the turbulence kinetic energy, and the six independent components of $f_{i j}$ are obtained by solving the six elliptic differential equations $\left(1-L^{2} \nabla^{2}\right) f_{i j}=f_{i j}^{h}$, with boundary conditions enabling the reproduction of the near-wall behavior of the redistribution term. These equations have then the purely geometrical effect, with a unique length scale. Their role is to enforce the redistribution terms to comply with their near-wall limiting behavior. It is, therefore, expected that the same effect could be reproduced with only one elliptic equation. A straightforward idea is to generalize the concept of scalar redistribution function $f$ used in the $\overline{v^{2}}-f$ model. A scalar function $f$ can be defined by $f=\phi_{k l}^{*} M_{k l}$, choosing an appropriate tensor $M_{k l}$ (e.g., the anisotropy tensor $a_{k l}$ ), and the pressure-strain term can be reconstructed from $\phi_{i j}^{*}=f N_{i j}$, where $N_{i j}$ is another well-chosen tensor. Unfortunately, no choice of $M_{k l}$ and $N_{i j}$ can rigorously ensure the exact reconstruction of $\phi_{i j}^{*}$ [in other words, the equation $\phi_{i j}^{*}=\left(\phi_{k l}^{*} M_{k l}\right) N_{i j}$ cannot be satisfied by any tensors $M_{k l}$ and $N_{i j}$, except obviously when $N_{i j}$ is set to $N_{i j}=\phi_{i j}^{*} /\left(\phi_{k l}^{*} M_{k l}\right)$, which is of no interest], and this type of model can hence be based only on an approximate reconstruction. A survey of different possibilities, through $a$ priori tests and computations in a channel flow, have led us to the conclusion that this approach cannot give correct predictions of the stress anisotropy in the near-wall region without using complex, nonlinear tensorial expressions for $M_{k l}$ and $N_{i j}$. Now, one of the major purposes of the elliptic relaxation approach is to avoid the use of such nonlinear formulations, and the appeal of such an approach, compared to nonlinear low-Reynolds-number models, diminishes if the level of nonlinearity is not reduced.

Therefore, in the present article, another approach is used based on a blending of near-wall and far-from-the-wall forms of the redistribution tensor, the ellipticity being preserved by solving an elliptic equation for the blending function $\alpha$. The model, called the elliptic blending model (EBM), preserves the main features of Durbin's Reynolds-stress model, but involves only one additional, scalar, elliptic equation, rather than six. We believe that this approach offers a reasonable compromise between simplicity and consistency with the physics.

The article is divided into five sections: after the Introduction, the constraints to be satisfied by a near-wall turbulence model are described. In the next section we outline the derivation of the model. A model validation in the channel flow at $\operatorname{Re}_{\tau}=590$, using direct numerical simulation (DNS) data, is described. Finally, some comments on the grid sensitivity issue are given.

\section{REYNOLDS-STRESS BUDGETS IN THE VICINITY OF THE WALL}

In order for a model to be as universal as possible, it must be based on true universal constraints (unlike the wall laws). In the near-wall region, the no-slip boundary condition and the incompressibility of the fluid impose the limiting behavior of the fluctuating quantities, and consequently, of the Reynolds stresses and their budgets. Reproducing these near-wall budgets is the only way to ensure a correct predic- 
TABLE I. Taylor-series expansions of the different terms of the budgets of the Reynolds stresses. The identities $\partial p_{0} / \partial x=2 \rho \nu a_{2}, \partial p_{0} / \partial z=2 \rho \nu c_{2}, p_{1}$ $=2 \rho \nu b_{2}$, and $p_{2}=3 \rho \nu b_{3}$ have been used, deriving from the fact that the fluctuating Navier-Stokes equations reduce in the near-wall region to $\partial p / \partial x_{i}$ $=\rho \nu \partial^{2} u_{i} / \partial y^{2}$.

\begin{tabular}{|c|c|c|c|c|c|c|c|}
\hline & $-\partial \overline{u_{i} u_{j}} / \partial t$ & $-C_{i j}$ & $D_{i j}^{\nu}$ & $D_{i j}^{T}$ & $\phi_{i j}^{*}$ & $P_{i j}$ & $-\varepsilon_{i j}$ \\
\hline$\overline{u^{2}}$ & $\mathcal{O}\left(y^{2}\right)$ & $\mathcal{O}\left(y^{3}\right)$ & $\begin{aligned} & 2 \nu \overline{a_{1}^{2}} \\
+ & 12 \nu \overline{a_{1} a_{2} y} \\
+ & \mathcal{O}\left(y^{2}\right) \\
& 12 \nu \overline{b_{2}^{2}} y^{2}\end{aligned}$ & $\mathcal{O}\left(y^{3}\right)$ & $\begin{array}{l}-4 \nu \overline{a_{1} a_{2}} y \\
+\mathcal{O}\left(y^{2}\right) \\
-4 \nu \overline{b_{2}^{2}} y^{2}\end{array}$ & $\mathcal{O}\left(y^{3}\right)$ & $\begin{array}{l}-2 \nu \overline{a_{1}^{2}} \\
-8 \nu \overline{a_{1} a_{2} y} \\
+\mathcal{O}\left(y^{2}\right) \\
-8 \nu \overline{b_{2}^{2}} y^{2}\end{array}$ \\
\hline$\overline{v^{2}}$ & $\mathcal{O}\left(y^{4}\right)$ & $\mathcal{O}\left(y^{5}\right)$ & $\begin{array}{c}+40 \nu \overline{b_{2} b_{3}} y^{3} \\
+\mathcal{O}\left(\frac{y^{4}}{)}\right) \\
2 \nu \overline{c_{1}^{2}}\end{array}$ & $\mathcal{O}\left(y^{5}\right)$ & $\begin{aligned}- & 16 \nu \overline{b_{2} b_{3}} y^{3} \\
& +\mathcal{O}\left(y^{4}\right)\end{aligned}$ & $\mathcal{O}\left(y^{5}\right)$ & $\begin{array}{c}-24 \nu \overline{b_{2} b_{3}} y^{3} \\
+\mathcal{O}\left(y^{4}\right) \\
-2 \nu \overline{c_{1}^{2}}\end{array}$ \\
\hline$\overline{w^{2}}$ & $\mathcal{O}\left(y^{2}\right)$ & $\mathcal{O}\left(y^{3}\right)$ & $\begin{array}{l}+12 \nu \overline{c_{1} c_{2} y} \\
+\mathcal{\mathcal { O } ( y ^ { 2 } )} \\
\quad 6 \nu \overline{a_{1} b_{2} y}\end{array}$ & $\mathcal{O}\left(y^{3}\right)$ & $\begin{array}{l}-4 \overline{c_{1} c_{2} y} \\
+\mathcal{O}\left(y^{2}\right) \\
-2 \nu \overline{a_{1} b_{2} y}\end{array}$ & $\mathcal{O}\left(y^{3}\right)$ & $\begin{array}{l}-8 \nu \overline{c_{1} c_{2} y} \\
+\mathcal{O}\left(y^{2}\right) \\
-4 \nu \overline{a_{1} b_{2} y}\end{array}$ \\
\hline$\overline{u v}$ & $\mathcal{O}\left(y^{3}\right)$ & $\mathcal{O}\left(y^{4}\right)$ & $\begin{array}{c}+\left(12 \nu \overline{a_{1} b_{3}}+12 \nu \overline{a_{2} b_{2}}\right) y^{2} \\
+\mathcal{O}\left(y^{3}\right) \\
2 \nu \overline{a_{1} c_{1}}\end{array}$ & $\mathcal{O}\left(y^{4}\right)$ & $\begin{array}{c}-\left(6 \nu \overline{a_{1} b_{3}}+4 \nu \overline{a_{2} b_{2}}\right) y^{2} \\
+\mathcal{O}\left(y^{3}\right)\end{array}$ & $\mathcal{O}\left(y^{4}\right)$ & $\begin{array}{c}-\left(6 \nu \overline{a_{1} b_{3}}+8 \nu \overline{a_{2} b_{2}}\right) y^{2} \\
+\mathcal{O}\left(y^{3}\right) \\
-2 \nu \overline{a_{1} c_{1}}\end{array}$ \\
\hline$\overline{u w}$ & $\mathcal{O}\left(y^{2}\right)$ & $\mathcal{O}\left(y^{3}\right)$ & $\begin{array}{c}+\left(6 \nu \overline{a_{1} c_{2}}+6 \nu \overline{a_{2} c_{1}}\right) y \\
+\mathcal{O}\left(y^{2}\right) \\
6 \nu \overline{b_{2} c_{1} y}\end{array}$ & $\mathcal{O}\left(y^{3}\right)$ & $\begin{array}{c}\left(-2 \nu \overline{a_{1} c_{2}}-2 \nu \overline{a_{2} c_{1}}\right) y \\
+\mathcal{O}\left(y^{2}\right) \\
-2 \nu \overline{b_{2} c_{1} y}\end{array}$ & $\mathcal{O}\left(y^{3}\right)$ & $\begin{array}{c}\left(-4 \nu \overline{a_{1} c_{2}}-4 \nu \overline{a_{2} c_{1}}\right) y \\
+\mathcal{O}\left(y^{2}\right) \\
-4 \nu \overline{b_{2} c_{1} y}\end{array}$ \\
\hline$\overline{v w}$ & $\mathcal{O}\left(y^{3}\right)$ & $\mathcal{O}\left(y^{4}\right)$ & $\begin{array}{c}+\left(12 \nu \overline{b_{3} c_{1}}+12 \nu \overline{b_{2} c_{2}}\right) y^{2} \\
+\mathcal{O}\left(y^{3}\right)\end{array}$ & $\mathcal{O}\left(y^{4}\right)$ & $\begin{array}{c}-\left(6 \nu \overline{b_{3} c_{1}}+4 \nu \overline{b_{2} c_{2}}\right) y^{2} \\
+\mathcal{O}\left(y^{3}\right)\end{array}$ & $\mathcal{O}\left(y^{4}\right)$ & $\begin{array}{c}-\left(6 \nu \overline{b_{3} c_{1}}+8 \nu \overline{b_{2} c_{2}}\right) y^{2} \\
+\mathcal{O}\left(y^{3}\right)\end{array}$ \\
\hline
\end{tabular}

tion of the wall-induced anisotropies in general configurations. Therefore, this is the main line followed in the derivation of the elliptic blending model. Note that free surfaces are not considered here and, consequently, modifications of the model are necessary to account for this type of boundary conditions.

We consider the general case of a wall in a turbulent incompressible flow: contrary to usual descriptions of the near-wall limiting behavior, the wall here is not necessarily plane and the flow may not be parallel to it. Let us focus on a certain point on the wall: the reference frame can, without any loss of generality, be chosen such that the $y$ direction be normal to the wall at this particular point, itself located at $y=0$. The mean velocities $U, V, W$ and the fluctuating velocities $u, v, w$ and pressure $p$ can be expressed as Taylorseries expansions in terms of $y$. The no-slip boundary condition leads to the canceling of the zeroth-order terms for the velocities, and the continuity equation to the canceling of the first-order terms for $V$ and $v$ :

$$
\begin{aligned}
& U=\mathcal{A}_{1}(x, z, t) y+\mathcal{A}_{2}(x, z, t) y^{2}+\mathcal{O}\left(y^{3}\right), \\
& V=\mathcal{B}_{2}(x, z, t) y^{2}+\mathcal{O}\left(y^{3}\right), \\
& W=\mathcal{C}_{1}(x, z, t) y+\mathcal{C}_{2}(x, z, t) y^{2}+\mathcal{O}\left(y^{3}\right), \\
& u=a_{1}(x, z, t) y+a_{2}(x, z, t) y^{2}+\mathcal{O}\left(y^{3}\right), \\
& v=b_{2}(x, z, t) y^{2}+\mathcal{O}\left(y^{3}\right), \\
& w=c_{1}(x, z, t) y+c_{2}(x, z, t) y^{2}+\mathcal{O}\left(y^{3}\right), \\
& p=p_{0}(x, z, t)+p_{1}(x, z, t) y+p_{2}(x, z, t) y^{2}+\mathcal{O}\left(y^{3}\right) .
\end{aligned}
$$

Note that the coefficients $A_{i}, B_{i}$, and $C_{i}$ are deterministic functions, whereas the coefficients $a_{i}, b_{i}, c_{i}$, and $p_{i}$ are stochastic variables.

The Taylor-series expansion of the Reynolds stresses is straightforward

$$
\begin{aligned}
& \overline{u^{2}}=\overline{a_{1}^{2}} y^{2}+2 \overline{a_{1} a_{2}} y^{3}+\mathcal{O}\left(y^{4}\right), \\
& \overline{v^{2}}=\overline{b_{2}^{2}} y^{4}+2 \overline{b_{2} b_{3}} y^{5}+\mathcal{O}\left(y^{6}\right), \\
& \overline{w^{2}}=\overline{c_{1}^{2}} y^{2}+2 \overline{c_{1} c_{2}} y^{3}+\mathcal{O}\left(y^{4}\right), \\
& \overline{u v}=\overline{a_{1} b_{2}} y^{3}+\left(\overline{a_{2} b_{2}}+\overline{a_{1} b_{3}}\right) y^{4}+\mathcal{O}\left(y^{5}\right), \\
& \overline{u w}=\overline{a_{1} c_{1}} y^{2}+\left(\overline{a_{1} c_{2}}+\overline{a_{2} c_{1}}\right) y^{3}+\mathcal{O}\left(y^{4}\right), \\
& \overline{v w}=\overline{b_{2} c_{1}} y^{3}+\left(\overline{b_{2} c_{2}}+\overline{b_{3} c_{1}}\right) y^{4}+\mathcal{O}\left(y^{5}\right) .
\end{aligned}
$$

The damping of the components involving $v$ is one of the major features a near-wall model must reproduce, in order to predict the two-component limit of turbulence.

The Reynolds-stress transport equations can be written as

$$
-\frac{\partial \overline{u_{i} u_{j}}}{\partial t}-C_{i j}+D_{i j}^{\nu}+D_{i j}^{T}+\phi_{i j}^{*}+P_{i j}-\varepsilon_{i j}=0
$$

where $C_{i j}, D_{i j}^{\nu}, D_{i j}^{T}, \phi_{i j}^{*}, P_{i j}$, and $\varepsilon_{i j}$ denote convection, viscous diffusion, turbulent diffusion, redistribution, production, and dissipation, respectively. Note that the term $\phi_{i j}^{*}$ is the pressure-velocity gradient correlation, which is purposely not decomposed into traceless and diffusive parts, since this splitting introduces near-wall behaviors that are 
difficult to reproduce. ${ }^{8,9}$ For convenience, $\phi_{i j}^{*}$ is called herein redistribution, even though it also contains a diffusive part.

The Taylor-series expansions of the terms in Eq. (3) are given in Table I. It is emphasized again that the general case is in focus here, i.e., all the derivatives with respect to $x, z$, and $t$ have been taken into account: they do not appear as the dominant orders (which are given explicitly in the table), but they are all contained in the $\mathcal{O}\left(y^{n}\right)$-for instance, the coefficient of the $y^{2}$ term in the expansion of $\varepsilon_{11}$ is $8 \nu \overline{a_{2}^{2}}$ $+12 \nu \overline{a_{1} a_{3}}+2 \nu\left(\partial a_{1} / \partial x\right)^{2}+2 \nu\left(\partial a_{1} / \partial z\right)^{2}$.

It can be seen that, for all the components, convection, turbulent diffusion, production, as well as the time derivative, are all negligible to the two first dominant orders in the wall region. Shown in Table I is that the behavior of $\overline{u_{i} u_{j}}$ in the vicinity of the wall is related to its limiting budget, which can be written, whatever the component, as

$$
\nu \frac{\partial^{2} \overline{u_{i} u_{j}}}{\partial y^{2}}-n(n+1) \nu \frac{\overline{u_{i} u_{j}}}{y^{2}}=\mathcal{O}\left(y^{n}\right),
$$

with $n=1$ for $\overline{u^{2}}, \overline{w^{2}}$ and $\overline{u w} ; n=2$ for $\overline{u v}$ and $\overline{v w}$; and $n$ $=3$ for $\overline{v^{2}}$. The solution of this second-order differential equation is

$$
\overline{u_{i} u_{j}}=B y^{n+1}+\frac{C}{y^{n}}+\mathcal{O}\left(y^{n+2}\right),
$$

where $C=0$ since $\overline{u_{i} u_{j}}$ is zero at the wall. Thus, it appears that the correct behavior of $\overline{u_{i} u_{j}}$ in $y^{n+1}$ can only be reproduced in computations by respecting the limiting behavior of $\phi_{i j}^{*}-\varepsilon_{i j}$ in $n(n+1) \nu \overline{u_{i} u_{j}} / y^{2}$.

The way the elliptic relaxation model proposed by Durbin $^{5}$ is expected to fulfill this requirement is (see, e.g., Durbin $^{5}$ or Manceau ${ }^{9}$ for details): first, by using the model

$$
\phi_{i j}^{*}-\varepsilon_{i j}=k f_{i j}-\frac{\overline{u_{i} u_{j}}}{k} \varepsilon
$$

and, second, by solving differential equations for $f_{i j}$

$$
f_{i j}-L^{2} \nabla^{2} f_{i j}=\frac{1}{k}\left(\phi_{i j}^{h}-\frac{2}{3} \varepsilon \delta_{i j}+\frac{\overline{u_{i} u_{j}}}{k} \varepsilon\right),
$$

called "elliptic relaxation equations" (other formulations of the elliptic relaxation operator were proposed $\left.{ }^{8,10,11}\right)$. The wall boundary conditions for $f_{i j}$ are

$$
\begin{aligned}
& f_{i j}^{w}=-\frac{20 \nu^{2}}{\varepsilon} \frac{\overline{u_{i} u_{j}}}{y^{4}} \text { for } f_{22}^{w}, f_{12}^{w}, \text { and } f_{23}^{w}, \\
& f_{i j}^{w}=-\frac{1}{2} f_{22}^{w} \text { for } f_{11}^{w} \text { and } f_{33}^{w}, \\
& f_{13}^{w}=0,
\end{aligned}
$$

and for $\varepsilon$

$$
\varepsilon=2 \nu \frac{k}{y^{2}} .
$$

Thus, in the near-wall budget of $\overline{v^{2}}$, using Eqs. (8) and (9), $\phi_{i j}^{*}-\varepsilon_{i j}$ becomes

$$
\begin{aligned}
\phi_{22}^{*}-\varepsilon_{22} & =k f_{22}-\frac{\overline{v^{2}}}{k} \varepsilon \\
& =-12 \nu \frac{\overline{\nu^{2}}}{y^{2}} .
\end{aligned}
$$

This complies with Eq. (4), and thus leads to the correct prediction of the limiting behavior of $\overline{v^{2}} \propto y^{4}$. In the case of $\overline{u^{2}}, \overline{w^{2}}$, and $\overline{u w}, k f_{i j}$ is negligible as compared with $\varepsilon_{i j}$, and, thus, the difference $\phi_{i j}^{*}-\varepsilon_{i j}$ reduces to $-\varepsilon \overline{u_{i} u_{j}} / k$, which ensures the correct limiting behavior $2 \nu \overline{u_{i} u_{j}} / y^{2}$. Hence, for these components Eq. (4) is satisfied, which ensures the correct prediction of $\overline{u^{2}}, \overline{w^{2}}$, and $\overline{u w} \propto y^{2}$.

However, a problem arises with $\overline{u v}$ and $\overline{v w}$. Indeed, for these components, $\phi_{i j}^{*}-\varepsilon_{i j}$ has the same behavior as for $\overline{v^{2}}$, and, accordingly, it leads to the predictions of $\overline{u v}$ and $\overline{v w}$ $\propto y^{4}$ instead of $y^{3}$. Hence, one may wonder why such boundary conditions are used for $f_{12}$ and $f_{23}$ : the reason ${ }^{8}$ is simply that no boundary condition can ensure a behavior in $y^{3}$ and that $y^{4}$ is preferable to $y^{2}$, in order that the turbulent shear stress remain negligible compared to the viscous shear stress in the near-wall budgets of the mean velocities.

To summarize, it is worth noting that the prediction of the two-component state of turbulence in the vicinity of the wall by the elliptic relaxation model is a consequence of the correct reproduction of $\phi_{i j}^{*}-\varepsilon_{i j}$ for the diagonal components, which is obtained by imposing appropriate boundary conditions to the elliptic equations for $f_{i j}$. This is obtained without spoiling the predictions in regions far from the wall, since the model degenerates to a standard high-Reynolds number model: $k f_{i j}-\varepsilon_{i j} \rightarrow \phi_{i j}^{h}-\frac{2}{3} \varepsilon \delta_{i j}$, where $\phi_{i j}^{h}$ can be any high-Reynolds number pressure-strain model, depending on the user's choice.

\section{DERIVATION OF THE ELLIPTIC BLENDING MODEL}

The main drawback of the Reynolds-stress ellipticrelaxation model is that it involves six additional equations for the independent components of the tensor $f_{i j}$, with boundary conditions (8), involving $1 / y^{4}$, which induce numerical stiffness. The aim of this paper is to provide a simpler model, while preserving the main qualities of the elliptic relaxation model, which are: the reproduction of the limiting wall behavior of $\phi_{i j}^{*}-\varepsilon_{i j}$ and, consequently, of the Reynolds stresses; the ellipticity of the model, which is necessary to account for the nonlocal blocking effect of the wall; ${ }^{9}$ the linearity of the model, or, more precisely, the fact that the use of the elliptic relaxation strategy does not increase the level of nonlinearity of the model (mainly due to the model $\phi_{i j}^{h}$ for the redistribution term). Our proposal is to model the redistribution term by

$$
\phi_{i j}^{*}=(1-k \alpha) \phi_{i j}^{w}+k \alpha \phi_{i j}^{h},
$$

and the dissipation by

$$
\varepsilon_{i j}=(1-A k \alpha) \frac{\overline{u_{i} u_{j}}}{k} \varepsilon+A k \alpha \frac{2}{3} \varepsilon \delta_{i j},
$$




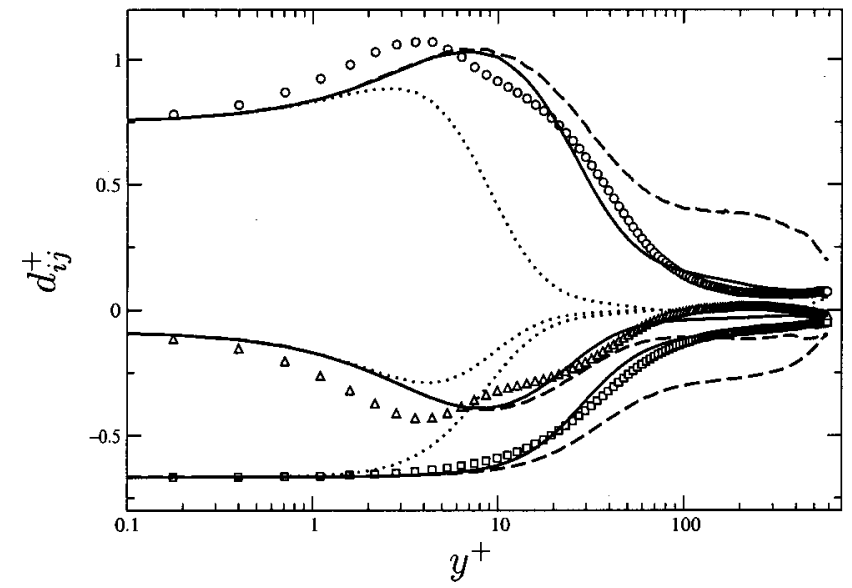

FIG. 1. A priori tests in a channel flow at $\operatorname{Re}_{\tau}=590$. DNS from Moser, Kim and Mansour (Ref. 16). Anisotropy of the dissipation tensor $d_{i j}=\varepsilon_{i j} / \varepsilon$ $-\frac{2}{3} \delta_{i j}$. Symbols: DNS $\left(\bigcirc d_{11} ; \square d_{22} ; \triangle d_{33}\right)$. --- $d_{i j}$ obtained using $\varepsilon_{i j}=\varepsilon \overline{u_{i} u_{j}} / k$. --- $d_{i j}$ obtained using $\varepsilon_{i j}=(1-k \alpha) \bar{\varepsilon} \overline{u_{i} u_{j}} / k+k \alpha \frac{2}{3} \varepsilon \delta_{i j}$. $-d_{i j}$ obtained using $\varepsilon_{i j}=(1-A k \alpha) \varepsilon \overline{u_{i} u_{j}} / k+A k \alpha \frac{2}{3} \varepsilon \delta_{i j}$.

where $A$ is Lumley's flatness parameter (see the Appendix). The ellipticity of the model is preserved by solving an elliptic differential equation for $\alpha$, similar to Eq. (7) solved for $f_{i j}$ in the elliptic relaxation model

$$
\alpha-L^{2} \nabla^{2} \alpha=\frac{1}{k}
$$

with the boundary condition $\alpha=0$ at the wall. The reason for using $1 / k$ as the source term of Eq. (14) and multiplying $\alpha$ by $k$ in Eqs. (12) and (13) is that it ensures a behavior of $k \alpha$ $\propto y^{3}$ in the vicinity of the wall, which makes the second term on the right-hand sides of Eqs. (12) and (13) negligible in this region. The factor $A$ has been introduced in the blending function used for $\varepsilon_{i j}$ in order to delay the transition from the near-wall form of $\varepsilon_{i j}$ to its far-from-the-wall form, as shown in Fig. 1. Note also that the solution of Eq. (14) exhibits a singularity at $y=0$ due to the behavior of $k \propto y^{2}$ : in order to suppress this singularity, $k$ is replaced in this equation by $\varepsilon T$, where, following Durbin, ${ }^{6} T$ is bounded by the Kolmogorov time scale

$$
T=\max \left(\frac{k}{\varepsilon}, C_{T}\left(\frac{\nu}{\varepsilon}\right)^{1 / 2}\right) .
$$

The length scale $L$ is also bounded by the Kolmogorov length scale

$$
L=C_{L} \max \left(\frac{k^{3 / 2}}{\varepsilon}, C_{\eta} \frac{\nu^{3 / 4}}{\varepsilon^{3 / 4}}\right) .
$$

In order to preserve the main feature of Durbin's model, which is the correct prediction of $\phi_{i j}^{*}-\varepsilon_{i j}$, the near-wall redistribution term $\phi_{i j}^{w}$ must be chosen in such a way that $\phi_{i j}^{*} / k$ tend to the values of $f_{i j}^{w}$ given in Eq. (8). This is achieved by choosing

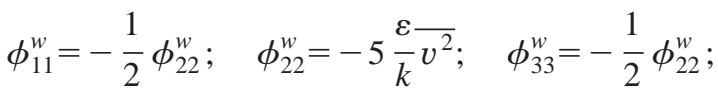

$$
\begin{aligned}
& \phi_{12}^{w}=-5 \frac{\varepsilon \overline{u v}}{k} ; \quad \phi_{13}^{w}=0 ; \quad \phi_{23}^{w}=-5 \frac{\varepsilon \bar{v} \bar{w} .}{.}
\end{aligned}
$$

Several important remarks about Eq. (17) should be made

(i) With the above wall values, the difference $\phi_{i j}^{*}-\varepsilon_{i j}$ has strictly the same behavior as that given by the elliptic relaxation model. The near-wall budgets of the Reynolds stresses are thus exactly the same as those described in Sec. II and the Reynolds stresses have the same limiting behavior.

(ii) For $\overline{v^{2}}$, the difference $\phi_{22}^{*}-\varepsilon_{22}$ is correctly reproduced near the wall but not each $\phi_{22}^{*}$ and $\varepsilon_{22}$ separately: this will not cause any problem since only their difference appears in the equations; however, this can be a source of discrepancies when comparing term by term predictions with the DNS data.

(iii) As for the elliptic relaxation model, the near-wall budgets of $\overline{u v}$ and $\overline{v w}$ are not correct, leading to behaviors $\propto y^{4}$ instead of $y^{3}$. This could have been avoided by choosing $\phi_{12}^{w}=-2 \frac{\varepsilon}{k} \overline{u v}$ and $\phi_{23}^{w}$ $=-2 \frac{\varepsilon}{\bar{v} w}$, allowing Eq. (4) to be satisfied and thus leading to the correct limiting behavior $\propto y^{3}$. This possibility has been investigated, but surprisingly it worsens the results in a channel flow. Therefore, Eq. (17) is preferred.

(iv) The values of $\phi_{11}^{w}$ and $\phi_{33}^{w}$ have been chosen such that $\phi_{i j}^{w}$ is traceless. This does not mean that what is modeled is the deviatoric part of the velocity-pressure gradient correlation (i.e., the pressure-strain term): if the velocity-pressure gradient correlation is split into pressure-strain and pressure diffusion, the latter also must be modeled in the near-wall region, since it becomes dominant in the budget of $\overline{v^{2}}$. ${ }^{9}$ Equation (4) is then valid only if the pressure diffusion is taken into account. Thus, what is modeled here, i.e., the term balancing $D_{i j}^{\nu}+\varepsilon_{i j}$, is definitely the velocitypressure gradient correlation. The choice for $\phi_{11}^{w}$ and $\phi_{33}^{w}$ is only made in order to ensure that the Reynoldsstress transport equation contracts to the standard $k$ equation (except for turbulent diffusion). This avoids the necessity of modifying the standard coefficients of the model too much, and in particular those of the $\varepsilon$ equation. This implies that $\phi_{11}^{w}$ and $\phi_{33}^{w}$ are not correctly modeled, but this is of minor importance, since they are small compared to $\varepsilon_{11}$ and $\varepsilon_{33}$, respectively.

Obviously, Eq. (17) needs to be written in a general, frame-independent form. To achieve this, it is necessary to identify somehow the direction normal to the wall. However, the use of a topological wall-normal vector must be avoided, since such a quantity is often not well defined in complex geometries. We propose here to use the fact that the gradient 


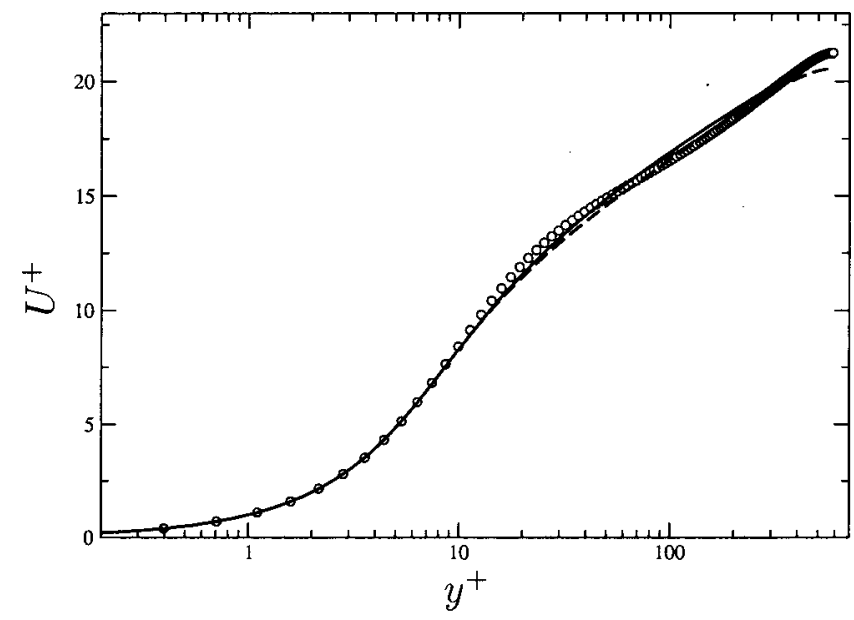

FIG. 2. Channel flow at $\operatorname{Re}_{\tau}=590$. Mean velocity. $\bigcirc$ DNS. --- Elliptic relaxation model. Elliptic blending model.

of the blending function $\alpha$ is normal to the wall in its vicinity, since the wall corresponds to the $\alpha=0$ isovalue contour. Thus, the vector

$$
\mathbf{n}=\frac{\boldsymbol{\nabla} \alpha}{\|\boldsymbol{\nabla} \alpha\|},
$$

can be used as a unit vector representing the "wall-normal" direction everywhere inside the domain. $\mathbf{n}$ cannot be defined only where $\|\nabla \alpha\|=0$, but this certainly happens only sufficiently far from the wall, where the factor $(1-k \alpha)$ makes the near-wall term negligible in Eq. (12). Some virtues of the use of this vector to identify the wall-normal direction can be noted: it avoids the discontinuity of the wall-normal vector across the bisector of a corner angle that appears with the usual geometrical definitions; it suppresses the need for determining the ambiguous "closest wall point," which can be multiply defined along a curved wall; it is sensitive to the curvature of the wall; it accounts automatically for all the

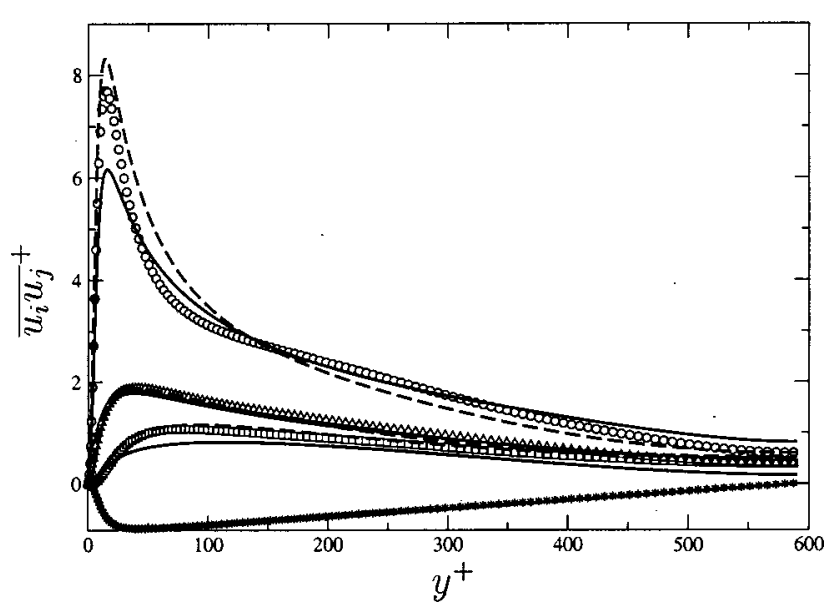

FIG. 3. Channel flow at $\operatorname{Re}_{\tau}=590$. Reynolds stresses. Symbols: DNS $\left(\bigcirc \overline{u^{2}} ; \square \overline{v^{2}} ; \triangle \overline{w^{2}} ; * \overline{u v}\right)$. --- Elliptic relaxation model. —_ Elliptic blending model.

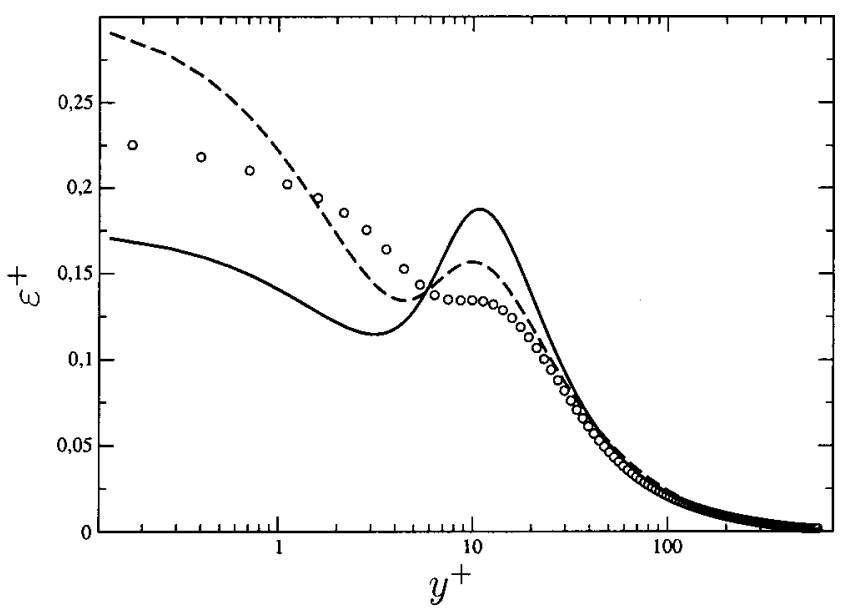

FIG. 4. Channel flow at $\operatorname{Re}_{\tau}=590$. Dissipation rate. $\bigcirc$ DNS. --- Elliptic relaxation model. —— Elliptic blending model.

walls present in the domain, contrary to the usual definitions, which favor the closest wall. Equation (17) can then be generalized to

$$
\begin{aligned}
\phi_{i j}^{w}= & -5 \frac{\varepsilon}{k}\left(\overline{u_{i} u_{k}} n_{j} n_{k}+\overline{u_{j} u_{k}} n_{i} n_{k}-\frac{1}{2} \overline{u_{k} u_{l}} n_{k} n_{l}\right. \\
& \left.\times\left(n_{i} n_{j}-\delta_{i j}\right)\right) .
\end{aligned}
$$

Concerning the far-from-the-wall part $\phi_{i j}^{h}$ any highReynolds-number model can be used. Two possibilities have been investigated: the Rotta+ IP model, ${ }^{12-14}$ and the Speziale, Sarkar, and Gatski model ${ }^{15}$ (SSG). The latter leads to somewhat better predictions and has been here selected as the preferred choice. Note that the coefficient $g_{3}^{*}$ (see the Appendix) has been set to 1.9 instead of 1.3: this has an influence mainly in the near-wall region, since it is in front of the square root of the second anisotropy invariant, which exhibits a peak in this region. The nonlinear return term $\left(g_{2}\right.$ term) can cause numerical stiffness and is often suppressed from the SSG model. However, it is necessary to predict correctly the return to isotropy problem (cf. Speziale et $a l .{ }^{15}$ ): hence, it is kept in the present model. If this term causes numerical difficulties, it can be suppressed: in that case, the coefficient $C_{\varepsilon 2}$ should be set to $C_{\varepsilon 2}=1.88$.

The model equations for $\overline{u_{i} u_{j}}$ are finally closed with the low-Re-number version of the transport equation for $\varepsilon$

$$
\begin{aligned}
\frac{\partial \varepsilon}{\partial t}+U_{k} \frac{\partial \varepsilon}{\partial x_{k}}= & \frac{C_{\varepsilon_{1}} P-C_{\varepsilon_{2}} \varepsilon}{T}+\frac{\partial}{\partial x_{l}}\left(\frac{C_{\mu}}{\sigma_{\varepsilon}} \overline{u_{l} u_{m}} T \frac{\partial \varepsilon}{\partial x_{m}}\right) \\
& +\nu \frac{\partial^{2} \varepsilon}{\partial x_{k} \partial x_{k}}+C_{\varepsilon_{3}} \nu \frac{k}{\varepsilon} \overline{u_{j} u_{k}}\left(\frac{\partial^{2} U_{i}}{\partial x_{j} \partial x_{l}}\right) \\
& \times\left(\frac{\partial^{2} U_{i}}{\partial x_{k} \partial x_{l}}\right),
\end{aligned}
$$

with the boundary condition (9). The term involving secondorder derivatives of the mean velocity is known to be numerically stiff and can require a very fine mesh in the buffer zone close to a wall, but can play an important physical role 

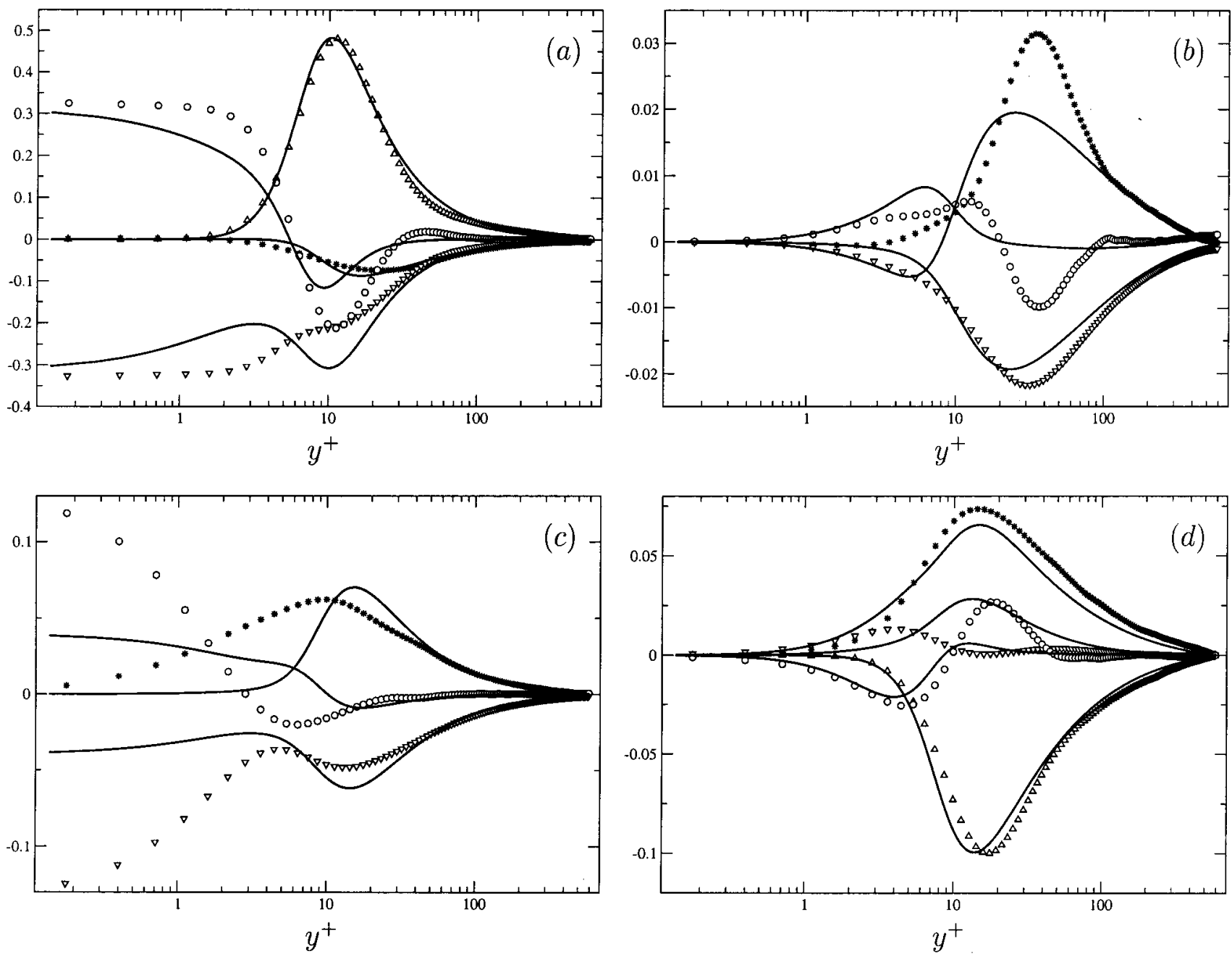

FIG. 5. Channel flow at $\operatorname{Re}_{\tau}=590$. Budgets of the Reynolds stresses. (a) $\overline{u^{2}}$; (b) $\overline{v^{2}}$; (c) $\overline{w^{2}}$; (d) $\overline{u v}$. Symbols: DNs $\left(\triangle\right.$ Production $P_{i j}$; $*$ Redistribution $\phi_{i j}^{*}$; $\nabla$ Dissipation $-\varepsilon_{i j}$; $\bigcirc$ Total diffusion $D_{i j}^{v}+D_{i j}^{T}$ ). $\_$Elliptic blending model.

in some situations, since it is a model for a term that appears in the exact $\varepsilon$ transport equation. An alternative version of the model is proposed in the Appendix, which does not use this term but a variable $C_{\varepsilon_{1}}$ coefficient: the latter version contains a bit less physics but is easier to compute.

The complete model equations are summarized in the Appendix.

\section{CHANNEL FLOW COMPUTATIONS}

The fully developed plane channel flow at $\operatorname{Re}_{\tau}=590$, for which a DNS database is available, ${ }^{16}$ is used to calibrate the model. The computations were performed with a simple 1D finite difference code, which allows one to impose the correct value of $\operatorname{Re}_{\tau}$. It is worth noting that the implementation of the model is very easy and that the numerical stiffness is considerably reduced compared to the elliptic relaxation model, whose boundary conditions (8) are major sources of numerical instability because of the denominator in $y^{4}$.

A very fine grid with 300 points across the flow is used, in order to avoid any numerical inaccuracy. The first nearwall point inside the domain is located at $y^{+} \simeq 0.1$, and the size of the largest cell in the center of the channel is $\Delta y^{+}$ $\simeq 5$. Note that such a small value of $y^{+}$for the first calculation point is not necessary: a value up to $y^{+} \simeq 3$ can be used without spoiling the predictions too much.

Profiles of mean velocity, Reynolds stresses, and dissipation rate, obtained by the elliptic blending model, are plotted in Figs. 2, 3, and 4, compared with the DNS and Durbin's elliptic relaxation model. ${ }^{5}$ It can be seen in Fig. 2 that the predictions of the mean velocity profile by both models are similar and reasonably close to the DNS, even though it appears that the slope in the logarithmic layer with both models is slightly underestimated. This seems to be an effect of the low Reynolds number, which may not have been fully captured with the model of the viscous effects-induced by imposing the Kolmogorov length scale as the lower bound, Eq. (16). However, Fig. 8 shows that, at high Reynolds number, the elliptic blending model reproduces correctly the logarithmic law.

Figure 3 shows that the anisotropy is globally well predicted. When comparing with the elliptic relaxation model, the peak of $\overline{u^{2}}$ is not as well captured, but its profile in the logarithmic layer is better reproduced. $\overline{v^{2}}$ is slightly underestimated too, but the profiles of $\overline{w^{2}}$ and $\overline{u v}$ are almost per- 


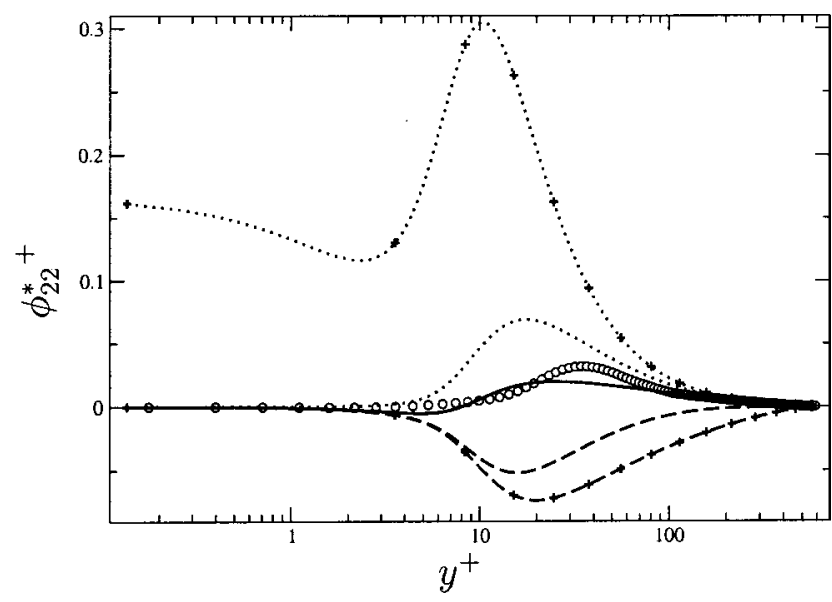

FIG. 6. Elliptic blending model: different contributions to $\phi_{22}^{*}$. $+\cdots+\phi_{22}^{h}$ (SSG model); $\cdots k \alpha \phi_{22}^{h} ;+---+\phi_{22}^{w} ;---(1-k \alpha) \phi_{22}^{w} ;-\phi_{22}^{*}$ $=(1-k \alpha) \phi_{22}^{w}+k \alpha \phi_{22}^{h} ; \bigcirc \phi_{22}^{*}$ from the DNs.

fect. It must be emphasized here that the elliptic blending strategy is able to make a high-Reynolds number model integrable down to the wall with only one additional equation, whereas the elliptic relaxation strategy uses six additional equations. The price to pay for the reduction of the complexity of the model is a loss of accuracy in the prediction of the anisotropies in the near-wall region. However, since the model is derived in such a way that the correct near-wall balances of the Reynolds-stress transport equations are satisfied, the crucial wall-blocking effect is preserved, which allows the prediction of the two-component limit of turbulence. It must also be noted that, in these computations, the SSG model has been used as the far-from-the-wall form $\phi_{i j}^{h}$ of the redistribution term, when the somewhat less elaborate Rotta+IP model has been used for the elliptic relaxation model.

In Fig. 4, it is observed that the dissipation rate $\varepsilon$ is well reproduced between $y^{+} \simeq 30$ and the center of the channel, but not below. Indeed, a peak around $y^{+}=10$ is predicted instead of a plateau, and the limiting value at the wall is

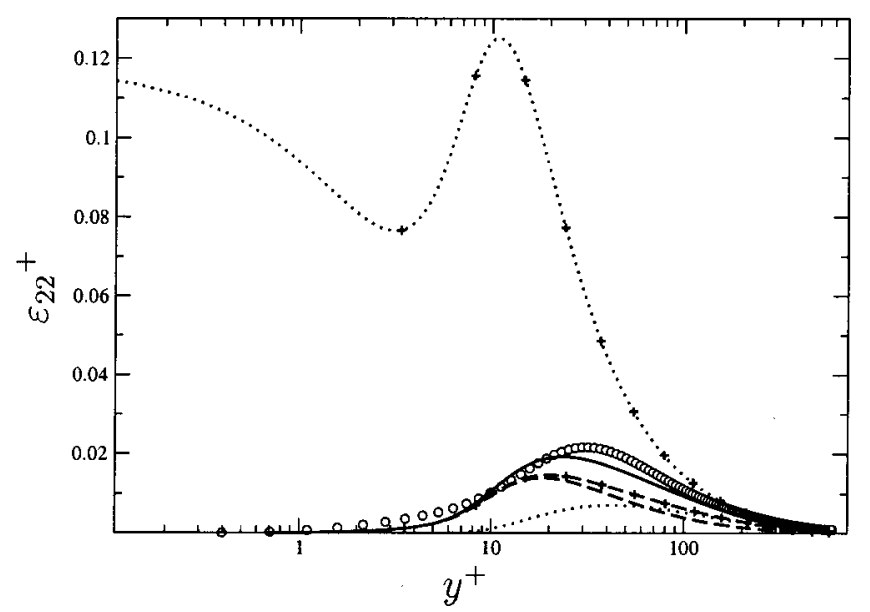

FIG. 7. Elliptic blending model: different contributions to $\varepsilon_{22} .+\cdots+\frac{2}{3} \varepsilon$; $\cdots A k \alpha \frac{2}{3} \varepsilon ; \quad+---+\varepsilon \overline{v^{2}} / k ; \quad--\quad(1-A k \alpha) \varepsilon \overline{v^{2}} / k ; \quad-\varepsilon_{22}$ $=(1-A k \alpha) \varepsilon \overline{v^{2}} / k+A k \alpha \frac{2}{3} \varepsilon ; \bigcirc \varepsilon_{22}$ from the DNS.

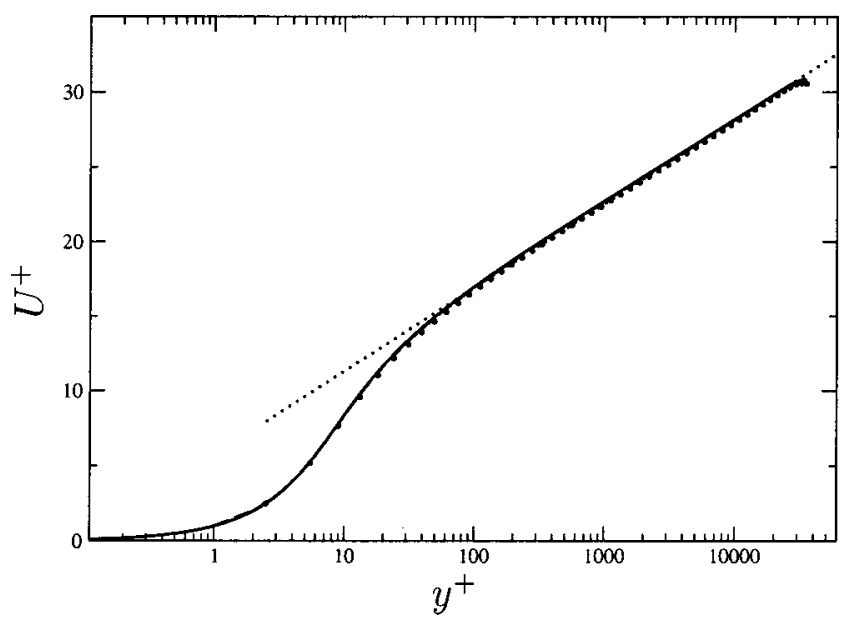

FIG. 8. Channel flow at $\mathrm{Re}=10^{6}$. Mean velocity profiles obtained with three different grids. $\longrightarrow$ Fine grid (500 points); --- Medium grid (100 points); Coarse grid (50 points, represented by the symbols); --- Log law: $U^{+}=\kappa^{-1} \ln y^{+}+5.7$, with $\kappa=0.41$.

underestimated by the elliptic blending model, and overestimated by the elliptic relaxation model. Note that, since the boundary condition (9) for $\varepsilon$ depends directly on $k$, this means that the second derivative of $k$ at the wall is slightly underestimated by the elliptic blending model, and overestimated by the elliptic relaxation model.

Figure 5 shows the budgets of the Reynolds stresses predicted by the elliptic blending model compared with the DNS. The budget of $\overline{u^{2}}$ [Fig. 5(a)] is fairly well reproduced: the dissipation is overestimated around $y^{+}=10$ and underestimated in the region below, as a consequence of the prediction of $\varepsilon$ (Fig. 4). This flaw is compensated by corresponding underestimation and overestimation, respectively, of the diffusion.

The budget of $\overline{v^{2}}$ [Fig. 5(b)] is not as good as that of $\overline{u^{2}}$. In particular, it is observed that very close to the wall $\left(y^{+}\right.$ $<2), \phi_{22}^{*}$ and $\varepsilon_{22}$ are not well predicted individually: as emphasized in Sec. III, only their difference is correctly re-

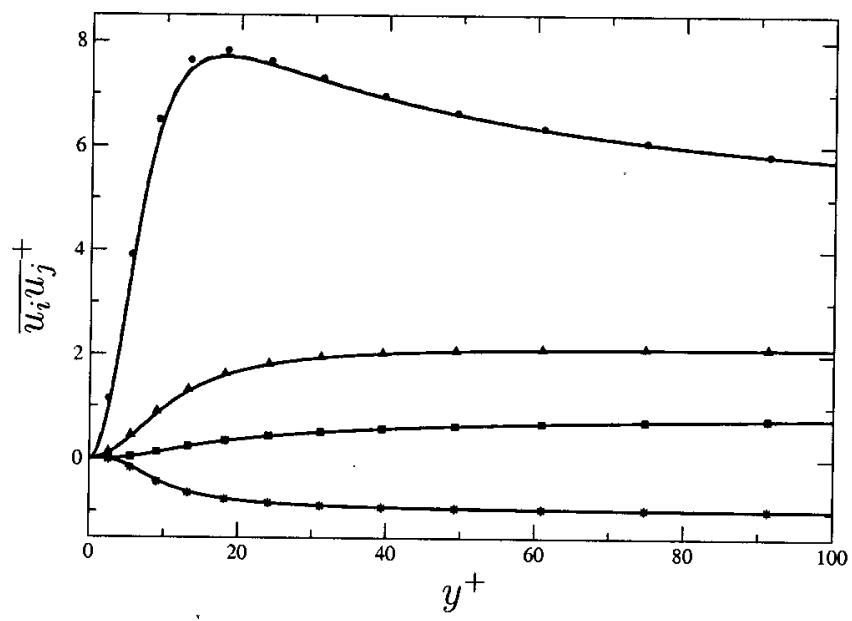

FIG. 9. Channel flow at $\mathrm{Re}=10^{6}$. Reynolds stress profiles obtained with three different grids. —— Fine grid (500 points); --- Medium grid (100 points); Symbols: Coarse grid (50 points, represented by the symbols, with: - $\overline{u^{2}} ; \mathbf{\square} \overline{v^{2}} ; \mathbf{\Delta} \overline{w^{2}} ; * \overline{u v}$ ). 
TABLE II. Characteristics of the grids used for the grid sensitivity analysis. The computation is performed for a channel flow at $\operatorname{Re}=10^{6}\left(\operatorname{Re}_{\tau} \simeq 35,000\right) . n$ denotes the number of points, and $y_{i}^{+}$the location of the $i$ th point of the grid.

\begin{tabular}{crllllll}
\hline \hline & $n$ & $y_{1}^{+}$ & $y_{2}^{+}$ & $y_{3}^{+}$ & $y_{4}^{+}$ & $\cdots$ & $y_{n}^{+}-y_{n-1}^{+}$ \\
\hline Fine grid & 500 & 0.10 & 0.21 & 0.31 & 0.42 & $\cdots$ & 330 \\
Medium grid & 100 & 1.0 & 2.1 & 3.3 & 4.6 & $\cdots$ & 1524 \\
Coarse grid & 50 & 2.5 & 5.5 & 9.0 & 13.2 & $\cdots$ & 2991 \\
\hline \hline
\end{tabular}

produced. In the region between $y^{+} \simeq 100$ and the center of the channel, all the contributions are well predicted. In the region below $y^{+} \simeq 100, \phi_{22}^{*}$ is not very well reproduced, but at least has the correct order of magnitude, unlike the one obtained with the homogeneous, high-Reynolds-number model alone (see Fig. 6). In the budget, this is again compensated by the diffusion term.

The budget of $\overline{w^{2}}$ [Fig. 5(c)] is well reproduced, except for the overestimation of the dissipation around $y^{+}=10$, already noted in the budget of $\overline{u^{2}}$, and its underestimation in the region below, compensated by the diffusion. The redistribution does not have the correct order of magnitude below $y^{+} \simeq 10$, as a consequence of the modeling of $\phi_{33}^{w}$ described in Sec. III.

In Fig. 5(d), it can be seen that the budget of $\overline{u v}$ is quite well reproduced: at least, the two dominant terms, production and redistribution, are very well predicted. Only the dissipation, and, as a compensation, the diffusion, which are of minor importance in this case, are not accurately predicted.

In summary, one can note that the budgets are in general satisfactory in the region very close to the wall $\left(y^{+}<10\right)$, where $\phi_{i j}^{*}$ and $\varepsilon_{i j}$ are dominated by their near-wall forms $\phi_{i j}^{w}$ and $\varepsilon u_{i} u_{j} / k$, and far from the wall $\left(y^{+}>100\right)$, where, in turn, the far-from-the-wall forms $\phi_{i j}^{h}$ and $\frac{2}{3} \varepsilon \delta_{i j}$ are dominant. In between, a buffer region exists, where $\phi_{i j}^{*}$ and $\varepsilon_{i j}$ experience a transition between their two forms. This behavior is detailed in Figs. 6 and 7 for $\phi_{22}^{*}$ and $\varepsilon_{22}$, respectively. It can be seen in Fig. 6 that the SSG model gives a correct prediction sufficiently far from the wall, but does not reproduce the damping of the redistribution very close to it. In the elliptic blending model, this damping is partly due to the factor $k \alpha$, and partly to the near-wall form $\phi_{22}^{w}$, which is negative, as is the $\phi_{22}^{*}$ given by the DNS. Figure 7 shows, similarly, that the isotropic model $\frac{2}{3} \varepsilon \delta_{i j}$ cannot reproduce the near-wall behavior of the dissipation tensor, and that the correct prediction of $\varepsilon_{\underline{22}}$ by the model is due to the blending of the near-wall form $\varepsilon u_{i} u_{j} / k$ and the isotropic form. Note also that the transition between the two forms occurs further from the wall for $\varepsilon_{22}$ than for $\phi_{22}^{*}$, as a consequence of the inclusion of the factor $A$ in the blending formula for the former. The location where the blending factor reaches the value 0.5 is indeed $y^{+} \simeq 30$ for $k \alpha ; y^{+} \simeq 180$ for $A k \alpha$.

\section{SOME COMMENTS ON GRID SENSITIVITY}

One of the reasons why near-wall models are criticized and, eventually, not used at all, is the fact that they often require a drastic refinement of the grid close to the wall when compared to models using wall functions. However, we found with our 1D code, using second-order finite differences, that the solution remains reasonably accurate when coarsening the grid. Figures 8 and 9 show results resolved in a channel flow at $\operatorname{Re}=10^{6}$. Three very different grids are used: the characteristics of these grids are summarized in Table II.

It is not claimed here that this possibility of using a coarse grid can be generalized to other numerical methods, like finite volumes, and to other flows, but the present results are, nevertheless, very encouraging since they contradict the usual belief that the wall region cannot be resolved without using a first grid point below $y^{+}=1$. This behavior of the model is probably due to the fact that the near-wall budgets (4) of the Reynolds stresses are satisfied, which induces, whatever the mesh, the correct behavior of the values at the first two points: for instance, for $\overline{u^{2}}$, the discretization of (4) with a second-order accurate finite difference scheme leads, at the dominant order, to $\overline{u^{2}}{ }_{2} / \overline{u^{2}}{ }_{1}=y_{2}^{2} / y_{1}^{2}$ (where indices 1 and 2 denote the values at the first and second near-wall points, respectively).

\section{CONCLUSION}

The issue of deriving near-wall models preserving a relative simplicity has been investigated. It has been shown that in order to predict the turbulence anisotropy in the vicinity of the wall (two-component limit), a model must reproduce the limiting behavior of $\phi_{i j}^{*}-\varepsilon_{i j}$, the difference between the redistribution and the dissipation. This requirement is fulfilled by the elliptic relaxation model of Durbin, ${ }^{5}$ but the penalty is an increase in the number of closure equations from seven to 13 , and numerically stiff boundary conditions for the six additional equations.

A new Reynolds-stress model, the elliptic blending model (EBM), has been proposed. This model has been derived on the basis of the elliptic relaxation model, but aimed at using only one additional closure equation (thus reducing the total number to eight), without sacrificing the main qualities of Durbin's Reynolds-stress model. It is noted that the six elliptic relaxation equations are somewhat redundant: they all provide a smooth transition between the near-wall and the far-from-the-wall forms of the model depending only on the geometry and the length scale, which is the same for all the components. Therefore, a similar effect can be obtained by using blending formulas for the redistribution $\phi_{i j}^{*}$ and the dissipation $\varepsilon_{i j}$, with blending factors $k \alpha$ and $A k \alpha$, respectively, going to zero at the wall and to 1 far from it. In order to preserve the nonlocal character of the model, which reflects the physical nonlocality of the blocking effect, the 
function $\alpha$ is defined as the solution of an elliptic differential equation, similar to the elliptic relaxation equations used in Durbin's model. The boundary condition for this equation at the wall is simply $\alpha=0$, which avoids numerical stiffness.

Tests in a channel flow show that the predictions with the new model are very similar to those of Durbin's model. The main difference is a less accurate prediction of the amplitude of the peak of the streamwise component of the Reynolds stress $\overline{u^{2}}$. The budgets of the Reynolds stresses are in general satisfactory.

These results are very encouraging, since they show that the elliptic blending model behavior in a channel flow is very similar to that of Durbin's model. Moreover, the strategy used for the near-wall region leads to only a moderate increase in complexity: it involves only one additional equation of elliptic type compared to standard Reynolds-stress models; it does not increase the level of nonlinearity (if a linear pressure-strain model is used as the far-from-the-wall formulation, the model of $\phi_{i j}^{*}$ is fully linear); it seems to allow the use of a reasonable grid density in the near-wall region.

We believe that the approach has good prospects of being applicable to a wide range of situations, since it is based on true universal physical constraints: the limiting behavior of the different terms of the Reynolds-stress budgets in the vicinity of the wall, which have been derived in a general case, and are thus valid even at separation and impinging points, in the presence of wall curvature, etc.

The model presented in this paper is certainly not a definitive, widely tested version, and an important effort is still necessary for testing and calibrating different modeling options. In the near future, computations of other canonical tests cases (backstep flow, impinging jet, square cylinder, etc.), as well as more complex flows will be performed for this purpose.

\section{APPENDIX: THE ELLIPTIC BLENDING MODEL}

\section{Equations}

$$
\begin{aligned}
& \frac{D \overline{u_{i} u_{j}}}{D t}=P_{i j}+D_{i j}^{\nu}+D_{i j}^{T}+\phi_{i j}^{*}-\varepsilon_{i j}, \\
& \frac{D \varepsilon}{D t}=\frac{C_{\varepsilon_{1}} P-C_{\varepsilon_{2}} \varepsilon}{T}+\frac{\partial}{\partial x_{l}}\left(\frac{C_{\mu}}{\sigma_{\varepsilon}} \overline{u_{l} u_{m}} T \frac{\partial \varepsilon}{\partial x_{m}}\right)+\nu \frac{\partial^{2} \varepsilon}{\partial x_{k} \partial x_{k}} \\
& +C_{\varepsilon_{3}} \nu \frac{k}{\varepsilon}-u_{j} u_{k}\left(\frac{\partial^{2} U_{i}}{\partial x_{j} \partial x_{l}}\right)\left(\frac{\partial^{2} U_{i}}{\partial x_{k} \partial x_{l}}\right) \text {, } \\
& \alpha-L^{2} \nabla^{2} \alpha=\frac{1}{\varepsilon T}, \\
& D_{i j}^{T}=\frac{\partial}{\partial x_{l}}\left(\frac{C_{\mu}}{\sigma_{k}} \overline{u_{l} u_{m}} T \frac{\partial \overline{u_{i} u_{j}}}{\partial x_{m}}\right), \\
& \phi_{i j}^{*}=(1-k \alpha) \phi_{i j}^{w}+k \alpha \phi_{i j}^{h}, \\
& \varepsilon_{i j}=(1-A k \alpha) \frac{\overline{u_{i} u_{j}}}{k} \varepsilon+A k \alpha \frac{2}{3} \varepsilon \delta_{i j},
\end{aligned}
$$

$$
\begin{aligned}
& A=1-\frac{9}{8}\left(a_{i j} a_{i j}-a_{i j} a_{j k} a_{k i}\right) ; \quad a_{i j}=\frac{\overline{u_{i} u_{j}}}{k}-\frac{2}{3} \delta_{i j}, \\
& \phi_{i j}^{h}=-\left(g_{1}+g_{1}^{*} \frac{P}{\varepsilon}\right) \varepsilon b_{i j}+g_{2} \varepsilon\left(b_{i k} b_{k j}-\frac{1}{3} b_{k l} b_{k l} \delta_{i j}\right) \\
& +\left(g_{3}-g_{3}^{*} \sqrt{b_{k l} b_{k l}}\right) k S_{i j}+g_{4} k\left(b_{i k} S_{j k}+b_{j k} S_{i k}\right. \\
& \left.-\frac{2}{3} b_{l m} S_{l m} \delta_{i j}\right)+g_{5} k\left(b_{i k} \Omega_{j k}+b_{j k} \Omega_{i k}\right), \\
& b_{i j}=\frac{\overline{u_{i} u_{j}}}{2 k}-\frac{1}{3} \delta_{i j} ; \quad S_{i j}=\frac{1}{2}\left(\frac{\partial U_{i}}{\partial x_{j}}+\frac{\partial U_{j}}{\partial x_{i}}\right) ; \\
& \Omega_{i j}=\frac{1}{2}\left(\frac{\partial U_{i}}{\partial x_{j}}-\frac{\partial U_{j}}{\partial x_{i}}\right), \\
& \phi_{i j}^{w}=-5 \frac{\varepsilon}{k}\left(\overline{u_{i} u_{k}} n_{j} n_{k}+\overline{u_{j} u_{k}} n_{i} n_{k}\right. \\
& \left.-\frac{1}{2} \overline{u_{k} u_{l}} n_{k} n_{l}\left(n_{i} n_{j}-\delta_{i j}\right)\right) \text {, } \\
& \mathbf{n}=\frac{\nabla \alpha}{\|\boldsymbol{\nabla} \alpha\|}, \\
& T=\max \left(\frac{k}{\varepsilon}, C_{T}\left(\frac{\nu}{\varepsilon}\right)^{1 / 2}\right) ; \quad L=C_{L} \max \left(\frac{k^{3 / 2}}{\varepsilon}, C_{\eta} \frac{\nu^{3 / 4}}{\varepsilon^{1 / 4}}\right) \text {. }
\end{aligned}
$$

\section{Coefficients}

$$
\begin{aligned}
& C_{\varepsilon_{1}}=1.4 ; \quad C_{\varepsilon_{2}}=1.85 ; \quad C_{\varepsilon_{3}}=0.55 ; \quad C_{\mu}=0.22 ; \\
& \sigma_{\varepsilon}=1.22 ; \\
& \sigma_{k}=1.0 ; \quad C_{L}=0.45 ; \quad C_{\eta}=80.0 ; \quad C_{T}=6.0 ; \\
& g_{1}=3.4 ; \quad g_{1}^{*}=1.8 ; \quad g_{2}=4.2 ; \quad g_{3}=0.8 ; \quad g_{3}^{*}=1.9 ; \\
& g_{4}=1.25 ; \quad g_{5}=0.4 .
\end{aligned}
$$

\section{Boundary conditions at the wall}

$$
U_{i}=0 ; \quad \overline{u_{i} u_{j}}=0 ; \quad \varepsilon=2 \nu \frac{k}{y^{2}} ; \alpha=0
$$

\section{Alternative version of the model for $\varepsilon$ equation}

For cases where the term involving $C_{\varepsilon_{3}}$ turns out to be unstable, $C_{\varepsilon_{3}}$ can be set to zero, and $C_{\varepsilon_{1}}$ set to

$$
C_{\varepsilon_{1}}=1.4\left(1 .+0.076(1-k \alpha) \sqrt{\frac{k}{\overline{u_{i} u_{j}} n_{i} n_{j}}}\right) \text {. }
$$

${ }^{1}$ S. V. Patankar and D. B. Spalding, Heat and Mass Transfer in Boundary Layers, (Morgan Grampian, London, 1967).

${ }^{2}$ J. L. Lumley, "Computational modeling of turbulent flows," Adv. Appl. Mech. 18, 123 (Academic, New York, 1978).

${ }^{3}$ T.-H. Shih and J. L. Lumley, "Second-order modeling of near-wall turbulence," Phys. Fluids 29, 971 (1986). 
${ }^{4}$ T. J. Craft and B. E. Launder, "A Reynolds stress closure designed for complex geometries," Int. J. Heat Fluid Flow 17, 245 (1996).

${ }^{5}$ P. A. Durbin, "A Reynolds stress model for near-wall turbulence," J. Fluid Mech. 249, 465 (1993).

${ }^{6}$ P. A. Durbin, "Near-wall turbulence closure modeling without 'damping functions,'," Theor. Comput. Fluid Dyn. 3, 1 (1991).

${ }^{7}$ K. Hanjalić, S. Jakirlić, and I. Hadžić, "Expanding the limits of 'equilibrium' second-moment turbulence closures," Fluid Dyn. Res. 29, 25 (1997).

${ }^{8}$ R. Manceau, Ph.D. thesis, Université de Nantes, France, 1999.

${ }^{9}$ R. Manceau, "Reproducing the blocking effect of the wall in one-point turbulence models," Proceedings of the European Congress Comput. Meth. Appl. Sciences and Engineering, Barcelona, Spain (2000).

${ }^{10}$ R. Manceau, M. Wang, and D. Laurence, "Inhomogeneity and anisotropy effects on the redistribution term in RANS modelling," J. Fluid Mech. 438, 307 (2001).
${ }^{11}$ R. Manceau and K. Hanjalić, "A new form of the elliptic relaxation equation to account for wall effects in RANS modelling," Phys. Fluids 12, $2345(2000)$

${ }^{12}$ J. C. Rotta, "Statistische Theorie nichthomogener Turbulenz," Z. Phys. 129, 547 (1951).

${ }^{13}$ D. Naot, A. Shavit, and M. Wolfshtein, "Two-point correlation model and the redistribution of Reynolds stresses," Phys. Fluids 16, 738 (1973).

${ }^{14}$ B. E. Launder, G. J. Reece, and W. Rodi, "Progress in the development of a Reynolds-stress turbulence closure," J. Fluid Mech. 68, 537 (1975)

${ }^{15}$ C. G. Speziale, S. Sarkar, and T. B. Gatski, "Modeling the pressure-strain correlation of turbulence: an invariant dynamical system approach," J. Fluid Mech. 227, 245 (1991).

${ }^{16}$ R. D. Moser, J. Kim, and N. N. Mansour, "Direct numerical simulation of turbulent channel flow up to $\operatorname{Re}_{\tau}=590$," Phys. Fluids 11, 943 (1999). 